\title{
Halldén Completeness for Relevant Modal Logics
}

\author{
Takahiro Seki
}

\begin{abstract}
Halldén completeness closely resembles the relevance property. To prove Halldén completeness in terms of Kripke-style semantics, the van Benthem-Humberstone theorem is often used. In relevant modal logics, the Halldén completeness of Meyer-Fuhrmann logics has been obtained using the van Benthem-Humberstone theorem. However, there remain a number of Halldén-incomplete relevant modal logics. This paper discusses the Halldén completeness of a wider class of relevant modal logics, namely, those with some Sahlqvist axioms.
\end{abstract}

\section{Introduction}

A propositional logic $\mathbf{L}$ is Halldén complete when, if $A \vee B$ is a theorem of $\mathbf{L}$, either $A$ is a theorem of $\mathbf{L}$ or $B$ is a theorem of $\mathbf{L}$ for all formulas $A$ and $B$ that do not have any propositional variables in common. As mentioned by Mares [4], Halldén completeness closely resembles the relevance property. Here, we say that a logic $\mathbf{L}$ has the relevance property when, if $A \rightarrow B$ is a theorem of $\mathbf{L}, A$ and $B$ have some propositional variables in common. If we think that $A \vee B$ and $\sim A \rightarrow B$ are "relatives," then the relevance property says that, when $A \vee B$ is a theorem of a relevant logic, $A$ and $B$ have some propositional variables in common. In this sense, the relevance property is a strengthened form of Halldén completeness.

It is well known that classical logic, intuitionistic logic, and modal logics such as $\mathbf{T}, \mathbf{S 4}$, and $\mathbf{S 5}$ are Halldén complete but that the modal logic $\mathbf{K}$ is not. In both relevant logics and relevant modal logics, there have been studies on Halldén completeness. Routley and Meyer [5] showed the Halldén completeness of the relevant modal logic NR using Routley-Meyer semantics. The generalization of the argument in [5] to a wider class of (nonmodal) relevant logics was discussed in Routley et al. [6, Section 5.7]. Furthermore, using the van Benthem-Humberstone theorem (see [10]), Mares [4] showed that all Meyer-Fuhrmann logics are Halldén complete

Received April 16, 2012; accepted January 22, 2013

2010 Mathematics Subject Classification: Primary 03B45; Secondary 03 B47

Keywords: Halldén completeness, relevant modal logics, Routley-Meyer semantics, van Benthem-Humberstone theorem, Sahlqvist formulas

(C) 2015 by University of Notre Dame 10.1215/00294527-2864334 
(Theorem 12) and also that there are Halldén-incomplete relevant modal logics that include $\square(A \vee B) \rightarrow \sim \square \sim A \vee \square B$ (Theorem 13). Note that Mares [4] dealt with modal logics based on the relevant logic $\mathbf{R}$.

The van Benthem-Humberstone theorem gives a sufficient condition for Halldén completeness. Because the theorem is stated in a frame-theoretical characterization, it is not necessarily clear from a logical viewpoint in which logics are Halldén complete. The Sahlqvist theorem is one of the fundamental results on the correspondence between modal formulas and frame postulates. Combining the Sahlqvist theorem and the van Benthem-Humberstone theorem, we expect to obtain a range of Halldén-complete modal logics with Sahlqvist axioms.

This paper extends the results in [4] and [6] to a wider class of relevant modal logics, namely, B.C $\square \diamond$ and its extensions using Sahlqvist axioms. B.C $\square \diamond$ was introduced as the basic relevant modal logic with the independent modal operators $\square$ and $\diamond$, and a Sahlqvist theorem for relevant modal logics was proven by Seki [7].

\section{Relevant Modal Logics}

The language of relevant modal logics consists of (i) propositional variables; (ii) logical connectives $\rightarrow, \wedge, \vee$, and $\sim$; (iii) modal operators $\square$ and $\diamond$; and (iv) a constant t. Formulas are defined in the usual way and are denoted by capital letters $A, B, C, D, E$. Prop and Wff will denote the set of all propositional variables and formulas, respectively. Furthermore, $\operatorname{Var}(A)$ will denote the set of propositional variables in $A \in \mathrm{Wff}$. We also introduce the following abbreviations:

$$
\triangleright A \stackrel{\text { def }}{=} \sim \diamond \sim A, \quad \diamond A \stackrel{\text { def }}{=} \square \sim A
$$

For $n \geq 1, \boldsymbol{\square}^{n} A$ represents $\boldsymbol{\square}_{n} \cdots \boldsymbol{\square}_{2} \boldsymbol{\square}_{1} A$, where $\boldsymbol{\square}_{i}(1 \leq i \leq n)$ denotes $\square$ or $\square$ and the $\boldsymbol{\square}_{i}$ are not necessarily the same; $\nabla^{n} A$ represents $\nabla_{n} \cdots \nabla_{2} \diamond_{1} A$, where $\diamond_{i}(1 \leq i \leq n)$ denotes $\diamond$ or $\diamond$ and the $\diamond_{i}$ are not necessarily the same. Moreover, let both $\square^{0} A$ and $\diamond^{0} A$ be $A$.

The relevant modal logic B. $\mathbf{C}_{\square \diamond}$ is defined as follows:

(a) Axioms

$$
\begin{aligned}
& \text { (A1) } A \rightarrow A, \\
& \text { (A2) } A \wedge B \rightarrow A, \\
& \text { (A3) } A \wedge B \rightarrow B, \\
& \text { (A4) }(A \rightarrow B) \wedge(A \rightarrow C) \rightarrow(A \rightarrow B \wedge C), \\
& \text { (A5) } A \rightarrow A \vee B, \\
& \text { (A6) } B \rightarrow A \vee B, \\
& \text { (A7) }(A \rightarrow C) \wedge(B \rightarrow C) \rightarrow(A \vee B \rightarrow C), \\
& \text { (A8) } A \wedge(B \vee C) \rightarrow(A \wedge B) \vee C, \\
& \text { (A9) } \sim A \rightarrow A, \\
& \text { (A10) t, } \\
& \text { (A11) } \square A \wedge \square B \rightarrow \square(A \wedge B), \\
& \text { (A12) } \diamond(A \vee B) \rightarrow \diamond A \vee \diamond B ;
\end{aligned}
$$


(b) Rules of inference

$$
\begin{aligned}
& \text { (R1) } \frac{A \rightarrow B \quad A}{B}, \quad\left(\text { R2) } \frac{A \quad B}{A \wedge B},\right. \\
& \text { (R3) } \frac{A \rightarrow B \quad C \rightarrow D}{(B \rightarrow C) \rightarrow(A \rightarrow D)}, \quad\left(\text { R4) } \frac{A \rightarrow \sim B}{B \rightarrow \sim A},\right. \\
& \text { (R5) } \frac{A}{\mathbf{t} \rightarrow A}, \quad(\text { R6 }) \frac{A \rightarrow B}{\square A \rightarrow \square B}, \\
& \text { (R7) } \frac{A \rightarrow B}{\diamond A \rightarrow \diamond B} .
\end{aligned}
$$

We provide the following lists of axioms and rules of inference:

$$
\begin{aligned}
& \text { (B1) } A \wedge(A \rightarrow B) \rightarrow B \text {, } \\
& \text { (B2) }(A \rightarrow \sim A) \rightarrow \sim A \text {, } \\
& \text { (B3) }(A \rightarrow(A \rightarrow B)) \rightarrow(A \rightarrow B) \text {, } \\
& \text { (B4) }(A \rightarrow B) \wedge(B \rightarrow C) \rightarrow(A \rightarrow C) \text {, } \\
& \text { (B5) }(A \rightarrow B) \rightarrow((B \rightarrow C) \rightarrow(A \rightarrow C)) \text {, } \\
& \text { (B6) }(A \rightarrow B) \rightarrow((C \rightarrow A) \rightarrow(C \rightarrow B)) \text {, } \\
& \text { (B7) } A \rightarrow((A \rightarrow B) \rightarrow B) \text {, } \\
& \text { (B8) }(A \rightarrow(B \rightarrow C)) \rightarrow(B \rightarrow(A \rightarrow C)) \text {, } \\
& \text { (B9) }(A \rightarrow(B \rightarrow C)) \rightarrow((A \rightarrow B) \rightarrow(A \rightarrow C)) \text {, } \\
& \text { (B10) }(A \rightarrow B) \rightarrow((A \rightarrow(B \rightarrow C)) \rightarrow(A \rightarrow C)) \text {, } \\
& \text { (B11) }(A \rightarrow B) \rightarrow((A \rightarrow C) \rightarrow(A \rightarrow B \wedge C)) \text {, } \\
& \text { (B12) } A \vee \sim A \text {, } \\
& \text { (B13) } A \rightarrow \sim(A \rightarrow \sim A) \text {, } \\
& \text { (B14) }(A \rightarrow \sim B) \rightarrow(B \rightarrow \sim A) \text {, } \\
& \text { (B15) } \square(A \rightarrow B) \rightarrow(\square A \rightarrow \square B) \text {, } \\
& \text { (B16) } \square(A \rightarrow B) \rightarrow(\square A \rightarrow \square B) \text {, } \\
& \text { (B17) } \square(A \rightarrow B) \rightarrow(\square A \rightarrow \square B) \text {, } \\
& \text { (B18) } \square(A \rightarrow B) \rightarrow(\square A \rightarrow \square B) \text {, } \\
& \text { (B19) } \square(A \rightarrow B) \rightarrow(\diamond A \rightarrow \diamond B) \text {, } \\
& \text { (B20) } \square(A \rightarrow B) \rightarrow(\diamond A \rightarrow \diamond B) \text {, } \\
& \text { (B21) } \square(A \rightarrow B) \rightarrow(\odot A \rightarrow \odot B) \text {, } \\
& \text { (B22) } \boxminus(A \rightarrow B) \rightarrow(\diamond A \rightarrow \diamond B) \text {, } \\
& \text { (D1) } \square A \rightarrow \diamond A \text {, } \\
& \text { (D2) } \square A \rightarrow \diamond A \text {, } \\
& \text { (D3) } \square A \rightarrow \diamond A \text {, }
\end{aligned}
$$

$$
\begin{aligned}
& \text { (Q1) } \frac{A}{(A \rightarrow B) \rightarrow B}, \quad(\mathrm{Q} 2) \frac{A}{\square A}, \\
& \text { (Q3) } \frac{A}{\square A}, \quad(\mathrm{Q} 4) \quad \frac{C \vee(A \rightarrow B) \quad C \vee A}{C \vee B}, \\
& \text { (Q5) } \frac{E \vee(A \rightarrow B) \quad E \vee(C \rightarrow D)}{E \vee((B \rightarrow C) \rightarrow(A \rightarrow D))}, \quad(\mathrm{Q} 6) \quad \frac{C \vee(A \rightarrow \sim B)}{C \vee(B \rightarrow \sim A)}, \\
& \text { (Q7) } \frac{C \vee(\sim A \rightarrow A)}{C \vee A}, \quad(\mathrm{Q} 8) \quad \frac{C \vee A}{C \vee \sim(A \rightarrow \sim A)}, \\
& \text { (Q9) } \frac{C \vee(A \rightarrow B)}{C \vee(\square A \rightarrow \square B)}, \quad \text { (Q10) } \frac{C \vee(A \rightarrow B)}{C \vee(\diamond A \rightarrow \diamond B)} .
\end{aligned}
$$

The rules of inference $(\mathrm{Q} 4)-(\mathrm{Q} 10)$ are called disjunctive rules. 
H1 denotes any logic obtained from B.C $\square \diamond$ by adding any set of axioms (B1)-(B22) and the rules of inference (Q1)-(Q10). H2 denotes any logic obtained from B. $\mathbf{C}_{\square \diamond}$ by adding any set of axioms (B1)-(B22) and the rules of inference (Q1)-(Q10) satisfying at least one of the following four conditions: (a) (B1) and (D1) are theorems; (b) (B1), (D2), and (D3) are theorems; (c) (B2) and (D1) are theorems; (d) (B2), (D2), and (D3) are theorems. Note that $\square A \rightarrow \diamond A$ is a theorem whenever (D1) is a theorem of $\mathbf{H} \mathbf{2}$.

It may be noted that the nonmodal reduct of $\mathbf{B} . \mathbf{C}_{\square \diamond}$ is the relevant logic $\mathbf{B}$. Let $\mathbf{L}+\Psi$ denote the logic obtained from $\mathbf{L}$ by adding a set $\Psi$ consisting of axioms and rules of inference. Then, $\mathbf{G}=\mathbf{B}+\{(\mathrm{B} 12)\}, \mathbf{T W}=\mathbf{B}+\{(\mathrm{B} 5),(\mathrm{B} 6),(\mathrm{B} 14)\}$, $\mathbf{T}=\mathbf{T W}+\{(\mathrm{B} 2),(\mathrm{B} 3)\}, \mathbf{E W}=\mathbf{T W}+\{(\mathrm{Q} 1)\}, \mathbf{E}=\mathbf{T}+\{(\mathrm{Q} 1)\}, \mathbf{R W}=\mathbf{E W}+$ $\{(\mathrm{B} 7)\}$, and $\mathbf{R}=\mathbf{E}+\{(\mathrm{B} 7)\}$.

Note that modal logics over relevant logics that have (B2) as a theorem, including $\mathbf{T}, \mathbf{E}$, and $\mathbf{R}$, are divided into $\mathbf{H 1}$ and $\mathbf{H} \mathbf{2}$ based on whether they contain the theorems (D1), (D2), or (D3). On the other hand, because neither (B1) nor (B2) is a theorem of $\mathbf{B}$ or of contractionless relevant logics such as $\mathbf{T W}, \mathbf{E W}$, and $\mathbf{R W}$, we will regard their modal logics as belonging to $\mathbf{H 1}$.

\section{Routley-Meyer Semantics}

We use $\&, \Rightarrow, \forall$, and $\exists$ to denote, respectively, conjunction, implication, universal, and existential quantifiers in the metalanguage. We omit parentheses by assuming that $\forall$ and $\exists$ bind more strongly than \& and that \& binds more strongly than $\Rightarrow$.

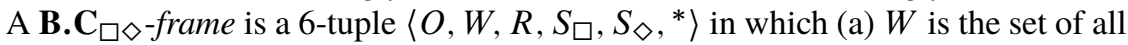
worlds, (b) $O$ is a nonempty subset of $W$, (c) $R$ is a ternary relation on $W$, (d) $S_{\square}$ and $S_{\diamond}$ are binary relations on $W$, and (e) ${ }^{*}$ is a unary operation on $W$. To simplify the notation, we define binary relations $\leq, S_{\varpi}$, and $S_{\odot}$ on $W$ as follows. For all $a, b \in W$ :

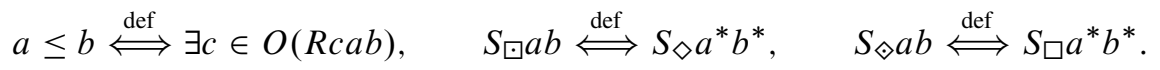

For a nonnegative integer $n$, the binary relations $S^{n}$ and $S^{n}$ on $W$, respectively, associated with $\boldsymbol{\square}^{n}\left(=\mathbf{\square}_{n} \cdots \boldsymbol{\square}_{2} \boldsymbol{\square}_{1}\right)$ and $\boldsymbol{\nabla}^{n}\left(=\boldsymbol{\nabla}_{n} \cdots \boldsymbol{}_{2} \boldsymbol{}_{1}\right)$, are defined as follows. For all $a, b \in W$ :

(i) $S_{a}^{0} a b$ iff $a \leq b$;

(ii) for $n \geq 1$,

$S_{\square}^{n} a b$ iff $\exists c_{1} \in W \cdots \exists c_{n-1} \in W\left(S_{\mathbf{\square}_{n}} a c_{n-1} \& \cdots \& S_{\mathbf{\square}_{2}} c_{2} c_{1} \& S_{\mathbf{\square}_{1}} c_{1} b\right)$;

(iii) $S_{\checkmark}^{0} a b$ iff $b \leq a$;

(iv) for $n \geq 1$,

$$
S_{\diamond}^{n} a b \text { iff } \exists c_{1} \in W \cdots \exists c_{n-1} \in W\left(S_{\diamond_{n}} a c_{n-1} \& \cdots \& S_{\diamond_{2}} c_{2} c_{1} \& S_{\diamond_{1}} c_{1} b\right) .
$$

A B.C $\square \diamond$-frame $\left\langle O, W, R, S_{\square}, S_{\diamond},{ }^{*}\right\rangle$ satisfies the following postulates. For all $a, b, c, d \in W$ :

(p1) $a \leq a$,

(p2) $a \leq b \& b \leq c \Rightarrow a \leq c$,

(p3) $a \leq b \& a \in O \Rightarrow b \in O$,

(p4) $a \leq b \&$ Rbcd $\Rightarrow$ Racd,

(p5) $a \leq b \& S_{\square} b c \Rightarrow S_{\square} a c$,

(p6) $a \leq b \& S_{\diamond a c} \Rightarrow S_{\diamond b c}$, 
(p7) $a \leq b \Rightarrow b^{*} \leq a^{*}$,

(p8) $a^{* *}=a$.

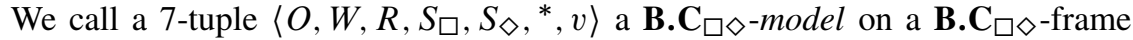
(or simply a B.C $\square \diamond$-model) $\mathfrak{F}=\left\langle O, W, R, S_{\square}, S_{\diamond},{ }^{*}\right\rangle$, where $\mathfrak{F}$ is a B.C. $\square \diamond$-frame and $v$ is a mapping from Prop $\times W$ to $\{\mathrm{t}, \mathrm{f}\}$, called a valuation on $\mathfrak{F}$, that satisfies the following hereditary condition. For all $a, b \in W$ and all $p \in$ Prop:

$$
a \leq b \& v(p, a)=\mathrm{t} \Rightarrow v(p, b)=\mathrm{t} .
$$

Given a B.C $\mathbf{C}_{\square \diamond-\text { model }}\left\langle O, W, R, S_{\square}, S_{\diamond},{ }^{*}, v\right\rangle$, we can define the interpretation $I$ associated with $v$. A mapping $I$ from $\mathrm{Wff} \times W$ to $\{\mathrm{t}, \mathrm{f}\}$ is defined inductively as follows. For $a \in W$ :

$$
\begin{aligned}
& \text { i. for any } p \in \operatorname{Prop}, I(p, a)=\mathrm{t} \text { iff } v(p, a)=\mathrm{t} \text {; } \\
& \text { ii. } I(A \wedge B, a)=\mathrm{t} \text { iff } I(A, a)=\mathrm{t} \& I(B, a)=\mathrm{t} \text {; } \\
& \text { iii. } I(A \vee B, a)=\mathrm{t} \text { iff } I(A, a)=\mathrm{t} \text { or } I(B, a)=\mathrm{t} \text {; } \\
& \text { iv. } I(A \rightarrow B, a)=\mathrm{t} \text { iff } \forall b \in W \forall c \in W(R a b c \& I(A, b)=\mathrm{t} \Rightarrow I(B, c)=\mathrm{t}) \text {; } \\
& \text { v. } I(\sim A, a)=\mathrm{t} \text { iff } I\left(A, a^{*}\right)=\mathrm{f} \text {; } \\
& \text { vi. } I(\square A, a)=\mathrm{t} \text { iff } \forall b \in W\left(S_{\square} a b \Rightarrow I(A, b)=\mathrm{t}\right) \text {; } \\
& \text { vii. } I(\diamond A, a)=\mathrm{t} \text { iff } \exists b \in W\left(S_{\diamond} a b \& I(A, b)=\mathrm{t}\right) \text {; } \\
& \text { viii. } I(\mathbf{t}, a)=\mathrm{t} \text { iff } a \in O \text {. }
\end{aligned}
$$

It is then easy to show the following. For all $a \in W$ and any nonnegative integer $n$ :

(a) $I(\square A, a)=\mathrm{t}$ iff $\forall b \in W\left(S_{\varpi} a b \Rightarrow I(A, b)=\mathrm{t}\right)$;

(b) $I(\diamond A, a)=\mathrm{t}$ iff $\exists b \in W\left(S_{\odot} a b \& I(A, b)=\mathrm{t}\right)$;

(c) $I\left(\boldsymbol{\square}^{n} A, a\right)=\mathrm{t}$ iff $\forall b \in W\left(S_{n}^{n} a b \Rightarrow I(A, b)=\mathrm{t}\right)$;

(d) $I\left(\diamond^{n} A, a\right)=\mathrm{t}$ iff $\exists b \in W\left(S^{n} a b \& I(A, b)=\mathrm{t}\right)$.

Then, by induction on the length of the formula $A$, we can show the following hereditary lemma.

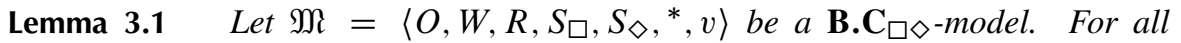
$a, b \in W$ and all $A \in \mathrm{Wff}$, if $a \leq b$ and $I(A, a)=\mathrm{t}$, then $I(A, b)=\mathrm{t}$.

Let $A \in$ Wff. We can say that (a) $A$ holds in a B.C $\mathbf{C}_{\square \diamond}$-model $\mathfrak{M}=\left\langle O, W, R, S_{\square}\right.$, $\left.S_{\diamond},{ }^{*}, v\right\rangle$ iff $I(A, a)=\mathrm{t}$ for every $a \in O$, and (b) $A$ is valid in a B.C $\square \diamond$-frame $\mathfrak{F}=\left\langle O, W, R, S_{\square}, S_{\diamond},{ }^{*}\right\rangle$ iff $A$ holds in every B.C. $\square \diamond$-model $\mathfrak{M}$ on $\mathfrak{F}$.

Let $\mathbf{L}$ be any extension of $\mathbf{B} . \mathbf{C}_{\square \diamond}$. We say that $\mathbf{L}$ is characterized by a class of B.C. $\mathbf{C}_{\square \diamond}$-frames $\boldsymbol{C}_{\mathrm{F}}$ if, for all $A \in \mathrm{Wff}, A$ is a theorem of $\mathbf{L}$ iff $A$ is valid in every $\mathfrak{F} \in \mathcal{C}_{\mathrm{F}}$. Any B.C $\mathbf{C}_{\square \diamond}$-frame in which all theorems of $\mathbf{L}$ are valid is called an $\mathbf{L}$-frame. $\mathbf{L}$-models (on $\mathbf{L}$-frames) are defined similarly to $\mathbf{B} . \mathbf{C}_{\square \diamond}$-models.

All the axioms and rules of inference we have mentioned have corresponding frame conditions. That is, if a logic $\mathbf{L}$ (over $\mathbf{B} . \mathbf{C}_{\square \diamond}$ ) contains an axiom or a rule of inference, then any $\mathbf{L}$-frame $\mathfrak{F}=\left\langle O, W, R, S_{\square}, S_{\diamond},{ }^{*}\right\rangle$ satisfies certain postulates. Here is a list of such correspondences. The postulate (q $i$ ) corresponds to axiom ( $\mathrm{B} i$ ) for $1 \leq i \leq 22$, the postulate ( $\mathrm{s} j$ ) corresponds to axiom $(\mathrm{D} j$ ) for $1 \leq j \leq 3$, and the postulate (rk) corresponds to the rule of inference $(\mathrm{Q} k)$ for $1 \leq k \leq 10$. For all $a, b, c, d, f \in W$ :
(q1) Raaa,
(q2) $\operatorname{Ra}^{*} a$,
(q3) $R a b c \Rightarrow \exists x \in W(R a b x \& R x b c)$,
(q4) $R a b c \Rightarrow \exists x \in W(\operatorname{Rab} x \& \operatorname{Raxc})$, 
(q5) $R a b c \& R c d f \Rightarrow \exists x \in W(\operatorname{Rad} x \& R b x f)$,

(q6) $R a b c \& R c d f \Rightarrow \exists x \in W(R b d x \& R a x f)$,

(q7) $R a b c \Rightarrow R b a c$,

(q8) $R a b c \& R c d f \Rightarrow \exists x \in W(\operatorname{Rad} x \& R x b f)$,

(q9) $R a b c \& R c d f \Rightarrow \exists x \in W \exists y \in W(\operatorname{Radx} \& R b d y \& R x y f)$,

(q10) $R a b c \& R c d f \Rightarrow \exists x \in W \exists y \in W(R a d x \& R b d y \& R y x f)$,

(q11) $R a b c \& R c d f \Rightarrow R a d f \& R b d f$,

(q12) $a \in O \Rightarrow a^{*} \leq a$,

(q13) $R a^{*} a a^{*}$,

(q14) $R a b c \Rightarrow R a c^{*} b^{*}$,

(q15) Rabc \& $S_{\square} c d \Rightarrow \exists x \in W \exists y \in W\left(S_{\square} a x \& S_{\square} b y \& R x y d\right)$,

(q16) $R a b c \& S_{\square} c d \Rightarrow \exists x \in W \exists y \in W\left(S_{\square} a x \& S_{\square} b y \& R x y d\right)$,

(q17) $R a b c \& S_{\square} c d \Rightarrow \exists x \in W \exists y \in W\left(S_{\square} a x \& S_{\square} b y \& R x y d\right)$,

(q18) $R a b c \& S_{\varpi} c d \Rightarrow \exists x \in W \exists y \in W\left(S_{\varpi} a x \& S_{\varpi} b y \& R x y d\right)$,

(q19) $R a b c \& S_{\diamond} b d \Rightarrow \exists x \in W \exists y \in W\left(S_{\square} a x \& S_{\diamond} c y \& R x d y\right)$,

(q20) $R a b c \& S_{\diamond} b d \Rightarrow \exists x \in W \exists y \in W\left(S_{\varpi} a x \& S_{\diamond} c y \& R x d y\right)$,

(q21) $R a b c \& S_{\diamond} b d \Rightarrow \exists x \in W \exists y \in W\left(S_{\square} a x \& S_{\diamond} c y \& R x d y\right)$,

(q22) $R a b c \& S_{\odot} b d \Rightarrow \exists x \in W \exists y \in W\left(S_{\varpi} a x \& S_{\odot} c y \& R x d y\right)$,

(s1) $\exists x \in W\left(S_{\square} a x \& S_{\diamond} a x\right)$,

(s2) $\exists x \in W\left(S_{\square} a x \& S_{\diamond} a x\right)$,

(s3) $\exists x \in W\left(S_{\diamond} a x \& S_{\varpi} a x\right)$,

(r1) $\exists x \in O(\operatorname{Raxa})$,

(r2) $a \in O \& S_{\square} a b \Rightarrow b \in O$,

(r3) $a \in O \& S_{\varpi} a b \Rightarrow b \in O$,

(r4) $a \in O \Rightarrow$ Raaa,

(r5) $a \in O \& R a b f \& R f c d \Rightarrow \exists x \in W \exists y \in W($ Racx \& Rbxy \& Rayd),

(r6) $a \in O \& R a b c \Rightarrow R a c^{*} b^{*}$,

(r7) $a \in O \Rightarrow R a^{*} a a^{*}$,

(r8) $a \in O \Rightarrow \operatorname{Raa}^{*} a$,

(r9) $a \in O \& R a b c \& S_{\square} c d \Rightarrow \exists x \in W\left(\operatorname{Raxd} \& S_{\square} b x\right)$,

(r10) $a \in O \& R a b c \& S_{\diamond} b d \Rightarrow \exists x \in W\left(\operatorname{Rad} x \& S_{\diamond} c x\right)$.

If H1 (or H2) is a logic in which all disjunctive rules can be derived from axioms and nondisjunctive rules, then we obtain completeness by the usual method of the canonical model (see Seki [7]). Otherwise, completeness can be proven by using the modified method of the canonical model, which is discussed in Seki [9].

Proposition 3.2 Let $\mathbf{L}$ be the logic $\mathbf{H 1}$ or $\mathbf{H 2}$. Then, $\mathbf{L}$ is characterized by a class of $\mathbf{L}$-frames.

\section{4 rp-Morphisms}

To adapt the van Benthem-Humberstone theorem to relevant modal logics, we need to define the following.

Let $\mathfrak{F}=\left\langle O, W, R, S_{\square}, S_{\diamond},{ }^{*}\right\rangle$ and $\mathfrak{F}^{\prime}=\left\langle O^{\prime}, W^{\prime}, R^{\prime}, S_{\square}^{\prime}, S_{\diamond}^{\prime},{ }^{* \prime}\right\rangle$ be B.C $\mathbf{C}_{\square \diamond-}$ frames. Then, a mapping $f$ from $W$ onto $W^{\prime}$ is a relevant p-morphism (rpmorphism) from $\mathfrak{F}$ to $\mathfrak{F}^{\prime}$ if the following conditions hold. For all $a, b, c \in W$ and $a^{\prime}, b^{\prime}, c^{\prime} \in W^{\prime}$ :

(m1) $f^{-1}\left(O^{\prime}\right)=O$,

(m2) $R a b c \Rightarrow R^{\prime} f(a) f(b) f(c)$, 
(m3) $R^{\prime} f(a) b^{\prime} c^{\prime} \Rightarrow \exists b \in W \exists c \in W\left(R a b c \& b^{\prime} \leq^{\prime} f(b) \& f(c) \leq^{\prime} c^{\prime}\right)$,

(m4) $S_{\square} a b \Rightarrow S_{\square}^{\prime} f(a) f(b)$,

(m5) $S_{\square}^{\prime} f(a) b^{\prime} \Rightarrow \exists b \in W\left(S_{\square} a b \& f(b) \leq^{\prime} b^{\prime}\right)$,

(m6) $S_{\diamond} a b \Rightarrow S_{\diamond}^{\prime} f(a) f(b)$,

(m7) $S_{\diamond}^{\prime} f(a) b^{\prime} \Rightarrow \exists b \in W\left(S_{\diamond} a b \& b^{\prime} \leq^{\prime} f(b)\right)$,

(m8) $f\left(a^{*}\right)=(f(a))^{* \prime}$.

This definition of rp-morphisms is due to Seki [8], where rp-morphisms are called frame morphisms and are defined for general frames. This definition is slightly different from that of Mares [4], but there is no fundamental difference between them.

Let $\mathfrak{F}^{\prime}=\left\langle O^{\prime}, W^{\prime}, R^{\prime}, S_{\square}^{\prime}, S_{\diamond}^{\prime},{ }^{* \prime}\right\rangle$ and $\mathfrak{F}^{\prime \prime}=\left\langle O^{\prime \prime}, W^{\prime \prime}, R^{\prime \prime}, S_{\square}^{\prime \prime}, S_{\diamond}^{\prime \prime},{ }^{* \prime \prime}\right\rangle$ be B.C $\square \diamond$-frames, and let $a \in W^{\prime}$ and $b \in W^{\prime \prime}$. Then, $(\mathfrak{F}, c)$ is an rp-morphic fusion of $\left(\mathfrak{F}^{\prime}, a\right)$ and $\left(\mathfrak{F}^{\prime \prime}, b\right)$ if and only if $\mathfrak{F}=\left\langle O, W, R, S_{\square}, S_{\diamond},{ }^{*}\right\rangle$ is a B.C. $\mathbf{C}_{\square \diamond}$-frame, $c \in W$, and there exist rp-morphisms $f^{\prime}$ from $\mathfrak{F}$ to $\mathfrak{F}^{\prime}$ and $f^{\prime \prime}$ from $\mathfrak{F}$ to $\mathfrak{F}$ ' such that $f^{\prime}(c)=a$ and $f^{\prime \prime}(c)=b$.

We now prove a relevant version of the van Benthem-Humberstone theorem. Though the theorem was essentially proven in [4, Theorem 6], we provide a proof to show that our definition of rp-morphisms also works well.

Theorem 4.1 If a logic $\mathbf{L}$ is characterized by a class of $\mathbf{B} . \mathbf{C}_{\square \diamond-f r a m e s}$ that are closed under rp-morphic fusion, then $\mathbf{L}$ is Halldén complete.

Proof Let $\mathbf{L}$ be a logic characterized by a class of $\mathbf{B} . \mathbf{C}_{\square \diamond}$-frames that are closed under rp-morphic fusion. Suppose that neither $A$ nor $B$ is a theorem of $\mathbf{L}$, where $\operatorname{Var}(A) \cap \operatorname{Var}(B)=\emptyset$. Then, there exist B.C $\mathbf{C}_{\square}$-frames $\mathfrak{F}^{\prime}=\left\langle O^{\prime}, W^{\prime}, R^{\prime}, S_{\square}^{\prime}\right.$, $\left.S_{\diamond}^{\prime},{ }^{* \prime}\right\rangle$ and $\mathfrak{F}^{\prime \prime}=\left\langle O^{\prime \prime}, W^{\prime \prime}, R^{\prime \prime}, S_{\square}^{\prime \prime}, S_{\diamond}^{\prime \prime},{ }^{* \prime \prime}\right\rangle$, valuations $v^{\prime}$ and $v^{\prime \prime}$ on $\mathfrak{F}^{\prime}$ and $\mathfrak{F}^{\prime \prime}$, respectively, and $a^{\prime} \in O^{\prime}, a^{\prime \prime} \in O^{\prime \prime}$ such that $I^{\prime}\left(A, a^{\prime}\right)=\mathrm{f}$ and $I^{\prime \prime}\left(B, a^{\prime \prime}\right)=\mathrm{f}$, where $I^{\prime}$ and $I^{\prime \prime}$ are the interpretations associated with $v^{\prime}$ and $v^{\prime \prime}$, respectively. Furthermore, there exists a frame $\mathfrak{F}=\left\langle O, W, R, S_{\square}, S_{\diamond},{ }^{*}\right\rangle$ and some $a \in W$ such that $(\mathfrak{F}, a)$ is an rp-morphic fusion of $\left(\mathfrak{F}^{\prime}, a^{\prime}\right)$ and $\left(\mathfrak{F}^{\prime \prime}, a^{\prime \prime}\right)$. Then, there are rpmorphisms $f^{\prime}$ from $\mathfrak{F}$ to $\mathfrak{F}^{\prime}$ and $f^{\prime \prime}$ from $\mathfrak{F}$ to $\mathfrak{F}^{\prime \prime}$ such that $f^{\prime}(a)=a^{\prime}$ and $f^{\prime \prime}(a)=a^{\prime \prime}$.

Define a valuation $v$ on $\mathfrak{F}$ as follows. For all $x \in W$ :

- for all $p \in \operatorname{Var}(A), v(p, x)=v^{\prime}\left(p, f^{\prime}(x)\right)$;

- for all $q \in \operatorname{Var}(B), v(q, x)=v^{\prime \prime}\left(q, f^{\prime \prime}(x)\right)$.

We show the following. For all $x \in W$, we have the following.

(1) For all formulas $C$ such that $\operatorname{Var}(C) \subseteq \operatorname{Var}(A), I(C, x)=I^{\prime}\left(C, f^{\prime}(x)\right)$.

(2) For all formulas $D$ such that $\operatorname{Var}(D) \subseteq \operatorname{Var}(B), I(D, x)=I^{\prime \prime}\left(D, f^{\prime \prime}(x)\right)$.

We prove (1) by induction on the length of $C$. The cases in which $C$ is of the form $p(\in \operatorname{Var}(A)), C_{1} \wedge C_{2}$, and $C_{1} \vee C_{2}$ are easily proven, so we restrict our attention to the following.

(a) $C$ is of the form $C_{1} \rightarrow C_{2}$. First, suppose that $I\left(C_{1} \rightarrow C_{2}, x\right)=\mathrm{t}$. To see $I^{\prime}\left(C_{1} \rightarrow C_{2}, f^{\prime}(x)\right)=\mathrm{t}$, suppose that $R^{\prime} f^{\prime}(x) b^{\prime} c^{\prime}$ and $I^{\prime}\left(C_{1}, b^{\prime}\right)=\mathrm{t}$. By (m3), there exist $b, c \in W$ such that $R x b c, b^{\prime} \leq^{\prime} f^{\prime}(b)$, and $f^{\prime}(c) \leq^{\prime} c^{\prime}$. By Lemma 3.1, we have $I^{\prime}\left(C_{1}, f^{\prime}(b)\right)=\mathrm{t}$, and hence $I\left(C_{1}, b\right)=\mathrm{t}$ by the inductive hypothesis. Then, $I\left(C_{2}, c\right)=\mathrm{t}$, and hence, using the inductive hypothesis again, $I^{\prime}\left(C_{2}, f^{\prime}(c)\right)=\mathrm{t}$. Thus, by Lemma 3.1, we have $I^{\prime}\left(C_{2}, c^{\prime}\right)=\mathrm{t}$, as desired. 
For the converse, suppose that $I^{\prime}\left(C_{1} \rightarrow C_{2}, f^{\prime}(x)\right)=\mathrm{t}$. To see $I\left(C_{1} \rightarrow C_{2}, x\right)=\mathrm{t}$, suppose that $R x b c$ and $I\left(C_{1}, b\right)=\mathrm{t}$. We have $R^{\prime} f^{\prime}(x) f^{\prime}(b) f^{\prime}(c)$ and $I^{\prime}\left(C_{1}, f^{\prime}(b)\right)=\mathrm{t}$ by $(\mathrm{m} 2)$ and the inductive hypothesis, respectively. Then, $I^{\prime}\left(C_{2}, f^{\prime}(c)\right)=\mathrm{t}$, and hence, by the inductive hypothesis, $I\left(C_{2}, c\right)=\mathrm{t}$, as desired.

(b) $C$ is of the form $\sim C_{1}$ :

$$
\begin{aligned}
I\left(\sim C_{1}, x\right)=\mathrm{t} & \text { iff } I\left(C_{1}, x^{*}\right)=\mathrm{f} \\
& \text { iff } I^{\prime}\left(C_{1}, f^{\prime}\left(x^{*}\right)\right)=\mathrm{f} \quad \text { (inductive hypothesis) } \\
& \text { iff } I^{\prime}\left(C_{1},\left(f^{\prime}(x)\right)^{* \prime}\right)=\mathrm{f} \quad(\mathrm{m} 8) \\
& \text { iff } I^{\prime}\left(\sim C_{1}, f^{\prime}(x)\right)=\mathrm{t} .
\end{aligned}
$$

(c) $C$ is of the form $\square C_{1}$. First, suppose that $I\left(\square C_{1}, x\right)=$ t. To see $I^{\prime}\left(\square C_{1}, f^{\prime}(x)\right)=\mathrm{t}$, suppose that $S_{\square}^{\prime} f^{\prime}(x) b^{\prime}$. By (m5), there exists $b \in W$ such that $S_{\square} x b$ and $f^{\prime}(b) \leq^{\prime} b^{\prime}$. Then, $I\left(C_{1}, b\right)=\mathrm{t}$, and hence $I^{\prime}\left(C_{1}, f^{\prime}(b)\right)=\mathrm{t}$ by the inductive hypothesis. Thus, by Lemma 3.1, we have $I^{\prime}\left(C_{1}, b^{\prime}\right)=\mathrm{t}$, as desired.

For the converse, suppose that $I^{\prime}\left(\square C_{1}, f^{\prime}(x)\right)=$ t. $\quad$ To see $I\left(\square C_{1}, x\right)=\mathrm{t}$, suppose that $S_{\square} x b$. By (m4), we have $S_{\square}^{\prime} f^{\prime}(x) f^{\prime}(b)$. Then, $I^{\prime}\left(C_{1}, f^{\prime}(b)\right)=\mathrm{t}$, and hence, by the inductive hypothesis, $I\left(C_{1}, b\right)=\mathrm{t}$, as desired.

(d) $C$ is of the form $\diamond C_{1}$. First, suppose that $I\left(\diamond C_{1}, x\right)=\mathrm{t}$. Then, there exists $b \in W$ such that $S_{\diamond x} x$ and $I\left(C_{1}, b\right)=\mathrm{t}$. We have $S_{\diamond}^{\prime} f^{\prime}(x) f^{\prime}(b)$ and $I^{\prime}\left(C_{1}, f^{\prime}(b)\right)=\mathrm{t}$ by $(\mathrm{m} 6)$ and the inductive hypothesis. Thus, $I^{\prime}\left(\diamond C_{1}, f^{\prime}(x)\right)=\mathrm{t}$, which is the desired result.

For the converse, suppose that $I^{\prime}\left(\diamond C_{1}, f^{\prime}(x)\right)=\mathrm{t}$. Then, there exists $b^{\prime} \in W^{\prime}$ such that $S_{\diamond}^{\prime} f^{\prime}(x) b^{\prime}$ and $I^{\prime}\left(C_{1}, b^{\prime}\right)=$ t. By $(\mathrm{m} 7)$, there exists $b \in W$ such that $S_{\diamond} x b$ and $b^{\prime} \leq^{\prime} f^{\prime}(b)$. We have $I^{\prime}\left(C_{1}, f^{\prime}(b)\right)=\mathrm{t}$ by Lemma 3.1, and hence $I\left(C_{1}, b\right)=\mathrm{t}$ by the inductive hypothesis. Therefore, $I\left(\diamond C_{1}, x\right)=\mathrm{t}$, which is the desired result.

(e) $C$ is of the form $\mathbf{t}$. Using $(\mathrm{m} 1)$,

$$
\begin{aligned}
I(\mathbf{t}, x)=\mathrm{t} & \text { iff } x \in O \\
& \text { iff } x \in f^{\prime-1}\left(O^{\prime}\right) \\
& \text { iff } f^{\prime}(x) \in O^{\prime} \\
& \text { iff } I^{\prime}\left(\mathbf{t}, f^{\prime}(x)\right)=\mathrm{t} .
\end{aligned}
$$

This completes the proof of (1). Claim (2) can be proven similarly.

Since $f^{\prime}(a)=a^{\prime}, f^{\prime \prime}(a)=a^{\prime \prime}, a^{\prime} \in O^{\prime}$, and $a^{\prime \prime} \in O^{\prime \prime}$, we have $a \in O$ by (m1). Since $I^{\prime}\left(A, f^{\prime}(a)\right)=\mathrm{f}$ and $I^{\prime}\left(B, f^{\prime \prime}(a)\right)=\mathrm{f}$, we have $I(A, a)=\mathrm{f}$ and $I(B, a)=\mathrm{f}$ by (1) and (2), respectively. Therefore, $I(A \vee B, a)=\mathrm{f}$. By assumption, $A \vee B$ is not a theorem of $\mathbf{L}$. This completes the proof.

\section{Products of Routley-Meyer Models}

We would like to know which logics are characterized by classes of frames that are closed under rp-morphic fusion. Mares [4] gave a partial answer to this question: RC and some of its extensions, the Meyer-Fuhrmann logics introduced in Section 6, are among them. 
Let $\mathfrak{F}^{\prime}=\left\langle O^{\prime}, W^{\prime}, R^{\prime}, S_{\square}^{\prime}, S_{\diamond}^{\prime},{ }^{* \prime}\right\rangle$ and $\mathfrak{F}^{\prime \prime}=\left\langle O^{\prime \prime}, W^{\prime \prime}, R^{\prime \prime}, S_{\square}^{\prime \prime}, S_{\diamond}^{\prime \prime},{ }^{* \prime \prime}\right\rangle$ be

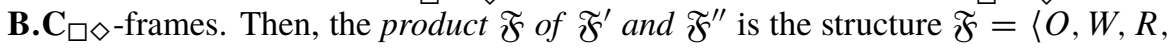
$\left.S_{\square}, S_{\diamond},{ }^{*}\right\rangle$ such that

- $W=\left\{\left\langle a_{1}, a_{2}\right\rangle \mid a_{1} \in W^{\prime} \& a_{2} \in W^{\prime \prime}\right\}\left(=W^{\prime} \times W^{\prime \prime}\right)$,

- $\left\langle a_{1}, a_{2}\right\rangle \in O \Longleftrightarrow a_{1} \in O^{\prime} \& a_{2} \in O^{\prime \prime}$ (i.e., $O=O^{\prime} \times O^{\prime \prime}$ ),

- $R\left\langle a_{1}, a_{2}\right\rangle\left\langle b_{1}, b_{2}\right\rangle\left\langle c_{1}, c_{2}\right\rangle \Longleftrightarrow R^{\prime} a_{1} b_{1} c_{1} \& R^{\prime \prime} a_{2} b_{2} c_{2}$,

- $S_{\square}\left\langle a_{1}, a_{2}\right\rangle\left\langle b_{1}, b_{2}\right\rangle \Longleftrightarrow S_{\square}^{\prime} a_{1} b_{1} \& S_{\square}^{\prime \prime} a_{2} b_{2}$,

- $S_{\diamond}\left\langle a_{1}, a_{2}\right\rangle\left\langle b_{1}, b_{2}\right\rangle \Longleftrightarrow S_{\diamond}^{\prime} a_{1} b_{1} \& S_{\diamond}^{\prime \prime} a_{2} b_{2}$,

- for all $\left\langle a_{1}, a_{2}\right\rangle \in W,\left\langle a_{1}, a_{2}\right\rangle^{*}=\left\langle a_{1}{ }^{* \prime}, a_{2}{ }^{* \prime \prime}\right\rangle$.

We say that a class $\mathcal{C}$ of $\mathbf{B} . \mathbf{C}_{\square \diamond}$-frames is strongly elementary if there is a set $\Phi$ of frame postulates that can be put in the form of a conjunction of conditions on $R$, $S_{\square}, S_{\diamond},{ }^{*}$, and $O$ implying a conjunction of conditions on $R, S_{\square}, S_{\diamond},{ }^{*}$, and $O$ such that, for every B.C $\mathbf{C}_{\square \diamond}$-frame $\mathfrak{F}, \mathfrak{F} \in \mathcal{C}$ iff $\mathfrak{F}$ satisfies each frame postulate in $\Phi$. The following lemma can then be easily verified.

Lemma 5.1 Every strongly elementary class of $\mathbf{B} . \mathbf{C}_{\square \diamond-f r a m e s}$ is closed under

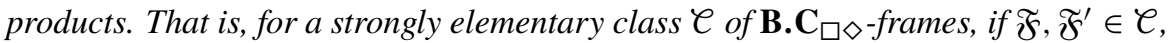
then the product of $\mathfrak{F}$ and $\mathfrak{F}^{\prime}$ is in $\mathcal{C}$.

Let $\mathfrak{F}=\left\langle O, W, R, S_{\square}, S_{\diamond},{ }^{*}\right\rangle$ be a frame. Then, we say:

- $\mathfrak{F}$ is $R$-serial iff, for all $a \in W, \exists x \in W \exists y \in W(\operatorname{Raxy})$;

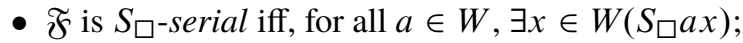

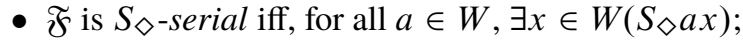

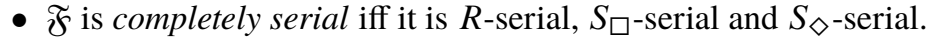

We notice that all $\mathbf{H 2}$-frames are completely serial because they satisfy (a) at least one of (q1) or (q2), which guarantees $R$-seriality, and (b) at least one of (s1) or both (s2) and (s3), which guarantees both $S_{\square-}$ and $S_{\diamond \text {-serialities. }}$

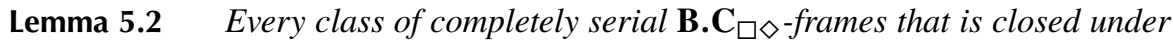
products is also closed under rp-morphic fusions.

Proof Let $\ell_{\mathrm{CSF}}$ be a class of completely serial B. $\mathbf{C}_{\square \diamond}$-frames that is closed under products. Furthermore, let $\mathfrak{F}=\left\langle O, W, R, S_{\square}, S_{\diamond},{ }^{*}\right\rangle$ be the product of $\mathfrak{F}^{\prime}=\left\langle O^{\prime}, W^{\prime}, R^{\prime}, S_{\square}^{\prime}, S_{\diamond}^{\prime},{ }^{* \prime}\right\rangle \in \mathcal{C}_{\mathrm{CSF}}$ and $\mathfrak{F}^{\prime \prime}=\left\langle O^{\prime \prime}, W^{\prime \prime}, R^{\prime \prime}, S_{\square}^{\prime \prime}, S_{\diamond}^{\prime \prime},{ }^{* \prime \prime}\right\rangle \in$ $\mathcal{C}_{\mathrm{CSF}}$. Then, $\mathfrak{F} \in \mathcal{C}_{\mathrm{CSF}}$. We define the mappings $f^{\prime}: W \rightarrow W^{\prime}$ by $f^{\prime}\left(\left\langle a_{1}, a_{2}\right\rangle\right)=a_{1}$ and $f^{\prime \prime}: W \rightarrow W^{\prime \prime}$ by $f^{\prime \prime}\left(\left\langle a_{1}, a_{2}\right\rangle\right)=a_{2}$. Then, we can easily see that both $f^{\prime}$ and $f^{\prime \prime}$ are rp-morphisms, but we present some typical examples of $f^{\prime}$.

(m1) Since $O^{\prime \prime} \neq \varnothing$, we have the following:

$$
\begin{aligned}
\left\langle a_{1}, a_{2}\right\rangle \in f^{\prime-1}\left(O^{\prime}\right) & \text { iff } f^{\prime}\left(\left\langle a_{1}, a_{2}\right\rangle\right) \in O^{\prime} \\
& \text { iff } a_{1} \in O^{\prime} \\
& \text { iff } a_{1} \in O^{\prime} \& a_{2} \in O^{\prime \prime} \\
& \text { iff }\left\langle a_{1}, a_{2}\right\rangle \in O .
\end{aligned}
$$

(m2) Suppose that $R\left\langle a_{1}, a_{2}\right\rangle\left\langle b_{1}, b_{2}\right\rangle\left\langle c_{1}, c_{2}\right\rangle$. By definition, $R^{\prime} a_{1} b_{1} c_{1}$ and $R^{\prime \prime} a_{2} b_{2} c_{2}$. Since $R^{\prime} a_{1} b_{1} c_{1}$, we have $R^{\prime} f^{\prime}\left(\left\langle a_{1}, a_{2}\right\rangle\right) f^{\prime}\left(\left\langle b_{1}, b_{2}\right\rangle\right) f^{\prime}\left(\left\langle c_{1}, c_{2}\right\rangle\right)$.

(m3) Suppose that $R^{\prime} f^{\prime}\left(\left\langle a_{1}, a_{2}\right\rangle\right) b_{1} c_{1}$. Then, $R^{\prime} a_{1} b_{1} c_{1}$. Since $\mathfrak{F}^{\prime \prime}$ is completely serial, there exist $b_{2}, c_{2} \in W^{\prime \prime}$ such that $R^{\prime \prime} a_{2} b_{2} c_{2}$. Therefore, $R\left\langle a_{1}, a_{2}\right\rangle\left\langle b_{1}, b_{2}\right\rangle\left\langle c_{1}, c_{2}\right\rangle, b_{1} \leq^{\prime} f^{\prime}\left(\left\langle b_{1}, b_{2}\right\rangle\right)$, and $f^{\prime}\left(\left\langle c_{1}, c_{2}\right\rangle\right) \leq^{\prime} c_{1}$. 
(m8) We have $f^{\prime}\left(\left\langle a_{1}, a_{2}\right\rangle^{*}\right)=f^{\prime}\left(\left\langle a_{1}{ }^{* \prime}, a_{2}{ }^{* \prime \prime}\right\rangle\right)=a_{1}{ }^{* \prime}=\left(f^{\prime}\left(\left\langle a_{1}, a_{2}\right\rangle\right)\right)^{* \prime}$.

Note that the proof of this lemma requires complete seriality. By Theorem 4.1 and Lemmas 5.1 and 5.2, we have the following.

Theorem 5.3 If a logic $\mathbf{L}$ is characterized by a strongly elementary class of completely serial $\mathbf{B} . \mathbf{C}_{\square \diamond-f r a m e s,}$ then $\mathbf{L}$ is Halldén complete.

\section{Halldén Completeness of $\mathrm{H} 1$}

Our proof of Theorem 5.3 naturally leads to an interest in knowing which logics are

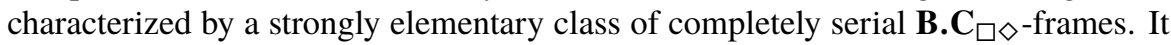
is clear that $\mathbf{H 2}$ is one such logic, since it is characterized by a class of $\mathbf{H 2}$-frames, which are strongly elementary, by Proposition 3.2, and all H2-frames are completely serial, as mentioned in Section 5. On the other hand, as indicated in [6], logics for which nondegenerate canonical modeling is not required are also characterized by a class of completely serial B. $\mathbf{C}_{\square \diamond}$-frames.

To see that $\mathbf{H 1}$ is characterized by a class of completely serial frames, we will examine its canonical model. Here, according to [7], we present key notions of the canonical model for a logic $\mathbf{H 1}$ in which all disjunctive rules can be derived from axioms and nondisjunctive rules. Let $a \subseteq \mathrm{Wff}$.

- $a$ is an H1-theory iff (a) if $A, B \in a$, then $A \wedge B \in a$, and (b) if $A \rightarrow B$ is a theorem of $\mathbf{H 1}$ and $A \in a$, then $B \in a$.

- An H1-theory $a$ is prime iff $A \vee B \in a$ implies $A \in a$ or $B \in a$.

- An H1-theory $a$ is regular iff $a$ contains all theorems of $\mathbf{H 1}$.

- An H1-theory $a$ is null iff there are no formulas in $a$.

- An H1-theory $a$ is universal iff every formula is in $a$.

Null and universal theories are called degenerate. For a logic $\mathbf{H 1}$ including disjunctive rules that cannot be derived from axioms and nondisjunctive rules, the third clause is replaced by the following.

- An H1-theory $a$ is regular iff (a) $a$ contains all theorems of H1, and (b) $a$ satisfies the following closure conditions for suitable disjunctive rules:

$$
\frac{C \vee A_{1} \quad\left(C \vee A_{2}\right)}{C \vee A_{3}}
$$

in H1; $C \vee A_{1} \in a$ (and $C \vee A_{2} \in a$ ) implies $C \vee A_{3} \in a$.

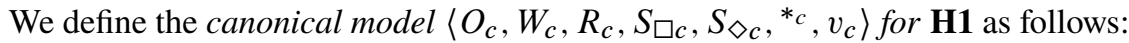

- $W_{c}$ is the set of all prime H1-theories;

- $O_{c}$ is the set of all regular prime H1-theories;

- $R_{c}$ is a ternary relation on $W_{c}$ defined by

$R_{c} a b c \Longleftrightarrow$ for all $A, B \in \mathrm{Wff}, \quad$ if $A \rightarrow B \in a$ and $A \in b$, then $B \in c$;

- $S_{\square c}$ is a binary relation on $W_{c}$ defined by

$$
S_{\square c} a b \Longleftrightarrow \text { for all } A \in \mathrm{Wff}, \quad \text { if } \square A \in a \text {, then } A \in b \text {; }
$$

- $S_{\diamond c}$ is a binary relation on $W_{c}$ defined by

$$
S_{\diamond_{c} a b} \Longleftrightarrow \text { for all } A \in \mathrm{Wff}, \quad \text { if } A \in b \text {, then } \diamond A \in a \text {; }
$$

- ${ }^{*_{c}}$ is a unary operation on $W_{c}$ defined by $a^{*_{c}}=\{A \in \mathrm{Wff} \mid \sim A \notin a\}$;

- $v_{c}$ is defined by $v_{c}(p, a)=\mathrm{t}$ iff $p \in a$, for all $p \in$ Prop and $a \in W_{c}$. 
If degenerate theories do not belong to $W_{c}$, then the canonical model is nondegenerate.

Concerning H1, Proposition 3.2 can be strengthened as follows.

Lemma 6.1 H1 is characterized by a class of completely serial H1-frames.

Proof Suppose that $A$ is not a theorem of H1. Furthermore, consider the canonical model $\left\langle O_{c}, W_{c}, R_{c}, S_{\square c}, S_{\diamond_{c}},{ }^{*_{c}}, v_{c}\right\rangle$ for $\mathbf{H 1}$ such that $I_{c}(A)=\mathrm{f}$. In H1, since nondegenerate canonical modeling is not required, we may assume that both $e_{c}$ and $u_{c}$ are in $W_{c}$, where $e_{c}=\emptyset$ and $u_{c}=\mathrm{Wff}$. Then, we have $R_{c} a u_{c} u_{c}, S_{\square c} a u_{c}$, and $S_{\diamond_{c}} a e_{c}$ for any $a \in W_{c}$, and hence the canonical frame for $\mathbf{H 1}$ is completely serial. Therefore, $A$ is not valid in a completely serial H1-frame.

Because a class of H1-frames is strongly elementary, we have the following by Lemma 6.1 and Theorem 5.3.

\section{Theorem 6.2 H1 is Halldén complete.}

The relevant modal logic $\mathbf{R C}$ is the $\diamond$ - and $\mathbf{t}$-free reduct of $\mathbf{R} . \mathbf{C}_{\square \diamond}$, where R.C $\mathbf{C}_{\square \diamond}=$ B.C $\square \diamond+\{(\mathrm{B} 2),(\mathrm{B} 5)$, (B6), (B8), (B14) $\}$. Since RC is regarded as a special case of $\mathbf{H 1}$, we have the following.

Corollary 6.3 (see [4]) $\quad$ RC is Halldén-complete.

Meyer-Fuhrmann schemes consist of (B15), (D2), $\square A \rightarrow A, \square A \rightarrow \square \square A$, $A \rightarrow \square \odot A, \diamond A \rightarrow \square \odot A$, and (Q2). Meyer-Fuhrmann logics (see [4]) are those obtained from RC by adding some or all of the Meyer-Fuhrmann schemes. Then, the following proposition holds.

Proposition 6.4 (see [4]) Every Meyer-Fuhrmann logic is Halldén complete.

To generalize the Meyer-Fuhrmann logics, consider the following axiom, called (ga):

$$
\diamond^{k} \mathbf{\square}^{l} A \rightarrow \mathbf{\square}^{m} \diamond^{n} A,
$$

for nonnegative integers $k, l, m, n$. The following frame postulate corresponds to (ga). For all $a, b, c \in W$ :

$$
S_{\triangleleft}^{k} a b \& S_{\square}^{m} a c \Rightarrow \exists x \in W\left(S_{\square}^{l} b x \& S_{\bullet}^{n} c x\right) .
$$

We will not give a proof of the completeness of $\mathbf{H 1}+\{(\mathrm{ga})\}$, but we remark that nondegenerate canonical modeling is not required for $\mathbf{H 1}+\{(\mathrm{ga})\}$. Therefore, we have the following, from which Proposition 6.4 follows as a corollary.

Theorem 6.5 Every logic $\mathbf{H 1}+\{(\mathrm{ga})\}$ is Halldén complete.

On the other hand, our discussion does not necessarily apply to logics obtained from H1 by adding the following axioms:

(C1) $A \rightarrow(B \rightarrow A)$,

(C2) $\square(A \vee B) \rightarrow \diamond A \vee \square B$.

The frame postulates corresponding to axioms (C1) and (C2) are (t1) and (t2), respectively. For all $a, b, c \in W$ :

(t1) $R a b c \Rightarrow a \leq c$,

(t2) $S_{\square} a b \Rightarrow \exists x \in W\left(x \leq b \& S_{\square} a x \& S_{\diamond} a x\right)$. 
First, consider the classical modal logic $\mathbf{K}$, where $\mathbf{K}=\mathbf{R} \mathbf{C}+\{(\mathrm{B} 15),(\mathrm{C} 1),(\mathrm{Q} 2)\}$. It is well known that $\mathbf{K}$ is not Halldén complete. It may seem that a proof of the Halldén completeness of $\mathbf{K}$ follows from our method, but because $\mathbf{K}$ requires nondegenerate canonical modeling in the sense of Routley-Meyer semantics, this expectation is not met. In fact, to see that the canonical model for $\mathbf{K}$ satisfies (t1), that is, $R_{c} a b c \Rightarrow a \leq_{c} c$, we must assume that $b$ is not a null $\mathbf{K}$-theory.

As another interesting example, the relevant modal logic $\mathbf{R K}$, where $\mathbf{R K}=\mathbf{R C}+$ $\{(\mathrm{B} 18),(\mathrm{Q} 2),(\mathrm{C} 2)\}$, is not Halldén complete (see [4]). It is remarkable that our canonical models are so-called tight, while the canonical model for RK discussed in Mares [2] is not. In the canonical model for $\mathbf{R K}, S_{\square c}$ is defined by

$$
\begin{aligned}
S_{\square c} a b \Longleftrightarrow & \text { (i) for all } A \in \mathrm{Wff} \text {, if } \square A \in a \text {, then } \odot A \in a \text {, and } \\
& \text { (ii) for all } A \in \mathrm{Wff} \text {, if } \square A \in a, \text { then } A \in b,
\end{aligned}
$$

and hence $S_{\square}$-seriality is not necessarily guaranteed. On the other hand, the canonical models for Meyer-Fuhrmann logics over $\mathbf{R K}+\{(\mathrm{D} 2)\}$ are tight (see Mares [3]), and these logics are Halldén complete (see [4] and Corollary 8.5 of this paper).

The following proposition shows the existence of Halldén-incomplete logics weaker than RK. This indicates the importance of (C2) in weaker relevant modal logics from the viewpoint of Halldén incompleteness.

Proposition 6.6 (see [4]) $\quad \mathbf{R C}+\{(\mathrm{C} 2)\}$ is not Halldén complete.

The proof in [4, Theorem 13] shows that (1) $(\sim \square p \vee \diamond p) \vee \square q$ is a theorem of $\mathbf{R C}+\{(\mathrm{C} 2)\}$ and that (2) neither $\sim \square p \vee \diamond p$ nor $\square q$ is a theorem of $\mathbf{R C}+\{(\mathrm{C} 2)\}$. Note that, as mentioned in [4], $\square p \vee \diamond p$ is a theorem of logics with axiom (D2). It is trivial that (2) holds for logics weaker than $\mathbf{R C}+\{(\mathrm{C} 2)\}$. Close examination of a proof of (1) reveals that both (B2) and (C2) play essential roles in ensuring that (1) holds.

Consider a generalized formula of (C2). We refer to formulas of the following form as $(\mathrm{gk})$ :

$$
\mathbf{\square}^{l}(A \vee B) \rightarrow \vee^{m} A \vee \mathbf{\square}^{n} B
$$

for nonnegative integers $l, m, n$. The following frame postulate corresponds to (gk). For all $a, b \in W$ :

$$
S_{\square}^{n} a b \Rightarrow \exists x \in W\left(x \leq b \& S_{\square}^{l} a x \& S_{\diamond}^{m} a x\right) .
$$

From the above observations, we have a generalization of Proposition 6.6.

Theorem 6.7 Let $\mathbf{L}$ be any logic $\mathbf{H 1}+\{(\mathrm{B} 2),(\mathrm{gk})\}$ or $\mathbf{H 1}+\{(\mathrm{B} 2),(\mathrm{ga}),(\mathrm{gk})\}$ that satisfies the following.

- There is no theorem of the form $\mathbf{\square}^{l} A \rightarrow \nabla^{n} A$, where $l+n>0$.

- The axiom (gk), $\mathbf{\square}^{l}(A \vee B) \rightarrow \vee^{m} A \vee \mathbf{\square}^{n} B$, satisfies $l+m>0$.

Then, $\mathbf{L}$ is not Halldén complete.

Note that none of (D1), (D2), or (D3) are theorems and that the formula $\mathbf{\square}^{l} A \rightarrow \Delta^{n} A$ is a special case of (ga).

When the necessitation rules $(\mathrm{Q} 2)$ and $(\mathrm{Q} 3)$ are assumed, we have the following.

Theorem 6.8 Let $\mathbf{L}$ be any logic $\mathbf{H 1}+\{(\mathrm{Q} 2),(\mathrm{Q} 3),(\mathrm{gk})\}$ or $\mathbf{H 1}+\{(\mathrm{Q} 2),(\mathrm{Q} 3)$, (ga), (gk)\} that satisfies the following. 
- $\mathbf{L}$ does not satisfy either (a) at least one of (B1) or (B2) is a theorem, or (b) the condition that there is no theorem of the form $\mathbf{\square}^{l} A \rightarrow \nabla^{n} A$, where $l+n>0$, (or both).

- The axiom (gk), $\mathbf{\square}^{l}(A \vee B) \rightarrow \diamond^{m} A \vee \mathbf{\square}^{n} B$, satisfies $m>0$.

Then, $\mathbf{L}$ is not Halldén complete.

Proof It is obvious that $(p \rightarrow p) \vee q$ is a theorem of $\mathbf{L}$, and so is $\mathbf{\square}^{l}((p \rightarrow p) \vee q)$ by (Q2) and (Q3). Using (gk), $\nabla^{m}(p \rightarrow p) \vee \boldsymbol{\square}^{n} q$ is a theorem of $\mathbf{L}$.

Take a one-world frame $\mathfrak{F}=\left\langle\{a\},\{a\}, R, S_{\square}, S_{\diamond},{ }^{*}\right\rangle$ that satisfies only Raaa. Note that $S_{\square}$ and $S_{\diamond}$ are empty relations, that is, neither $S_{\square} a a$ nor $S_{\diamond} a a$ hold, and that $a^{*}=a$. We can easily see that $\mathfrak{F}$ is an $\mathbf{L}$-frame and that $I\left(\boldsymbol{\nabla}^{m}(p \rightarrow p), a\right)=\mathrm{f}$ for any valuation $v$ on $\mathfrak{F}$. Since $\mathbf{L}$ is characterized by a class of $\mathbf{L}$-frames, $\nabla^{m}(p \rightarrow p)$ is not a theorem of $\mathbf{L}$.

Furthermore, take another one-world frame $\mathfrak{F}^{\prime}=\left\langle\{b\},\{b\}, R^{\prime}, S_{\square}^{\prime}, S_{\diamond}^{\prime},{ }^{* \prime}\right\rangle$ that satisfies $R^{\prime} b b b, S_{\square}^{\prime} b b$, and $S_{\diamond}^{\prime} b b$. Then, we can easily see that $\mathfrak{F}^{\prime}$ is an $\mathbf{L}$-frame and that $I^{\prime}\left(\boldsymbol{\square}^{n} q, b\right)=\mathrm{f}$ when a valuation $v^{\prime}$ on $\mathfrak{F}^{\prime}$ is defined by $v^{\prime}(q, b)=\mathrm{f}$. Thus, $\mathbf{\square}^{n} q$ is not a theorem of $\mathbf{L}$.

Since $\operatorname{Var}\left(\nabla^{m}(p \rightarrow p)\right) \cap \operatorname{Var}\left(\boldsymbol{\square}^{n} q\right)=\emptyset, \mathbf{L}$ is not Halldén complete.

\section{A Sahlqvist Theorem}

A Sahlqvist theorem is one of the fundamental results of correspondence and Kripke completeness, and it was proven for relevant modal logics by Seki [7]. In this section, we provide a brief explanation of a full version of the Sahlqvist formulas in order to explain the necessity of the restrictions imposed in the next section.

A formula $A$ is positive if it can be constructed using no connectives other than $\wedge, \vee, \square, \diamond, \square$, and $\odot$. A positive formula of the form $\square^{m_{1}} p_{1} \wedge \cdots \wedge \square^{m_{k}} p_{k}$, in which the propositional variables $p_{1}, \ldots, p_{k}$ are not necessarily distinct, is called a strongly positive formula. A given formula $A$ is negative (in a logic $\mathbf{L}$ ) if it is equivalent in $\mathbf{L}$ to $\sim B$ for a positive formula $B$. A modal formula $A$ is untied (in $\mathbf{L}$ ) if it can be constructed from strongly positive formulas and negative formulas (in $\mathbf{L}$ ) by using only $\wedge, \diamond$, and $\diamond$. A formula $A$ is called Sahlqvist if it is a conjunction of the form $\mathbf{\square}^{k}(B \rightarrow C)$, where $k \geq 0, B$ is untied in $\mathbf{L}$, and $C$ is positive.

In order to state a Sahlqvist theorem, we first establish some notions. It should be noted that the logic obtained from B. $\mathbf{C}_{\square \diamond}$ by adding a Sahlqvist axiom, for example, $p \wedge \sim p \rightarrow q$, is incomplete with respect to frames in the usual sense. Thus, to obtain frames for which the logic will be complete, we introduce enlarged frames with the null world $e$ and the universal world $u$. An enlarged $\mathbf{B} . \mathbf{C}_{\square \diamond-f r a m e}$ $\left\langle O, W, R, S_{\square}, S_{\diamond},{ }^{*}, e, u\right\rangle$ is obtained from a $\mathbf{B} . \mathbf{C}_{\square \diamond}$-frame $\left\langle O, W, R, S_{\square}, S_{\diamond},{ }^{*}\right\rangle$ by adding the elements $e, u \in W$. The enlarged frame then satisfies the following definition and postulates. For all $a, b \in W$ :

$$
\begin{array}{ll}
\text { (ep1) } e \neq u, & \text { (ep2) } e^{*}=u, \\
\text { (ep3) } \quad \text { Ruab } \Rightarrow a=e \text { or } b=u, & \text { (ep4) Reue, } \\
\text { (ep5) } S_{\square} u a \Rightarrow a=u, & \text { (ep6) } S_{\square} e e, \\
\text { (ep7) } S_{\diamond e a \Rightarrow a=e,} & \text { (ep8) } S_{\diamond u u} .
\end{array}
$$

The following is called a Sahlqvist theorem. Note that we use Routley-Meyer semantics with the null and universal worlds. 


\section{Proposition 7.1 (see [7])}

(1) Let $\mathbf{L}_{0}$ be the $\mathbf{H 1}$ or $\mathbf{H 2}$ logics defined above. For any Sahlqvist formula $A$, there exists a first-order formula $\varphi(a)$ in the predicates $O, R, S_{\square}, S_{\diamond}$, *, and the constants $e, u$ having $a$ as its only free variable and in which the following holds for every descriptive $\mathbf{L}_{0}$-frame or enlarged $\mathbf{L}_{0}$-frame $\mathfrak{F}$ and every $a \in W$ :

$$
(\mathfrak{F}, a) \models A \text { iff } \mathfrak{F} \text { satisfies } \varphi(a),
$$

where $(\mathfrak{F}, a) \models A$ means that $I(A, a)=\mathrm{t}$ under any valuation on $\mathfrak{F}$.

(2) Let $\mathbf{L}$ be a logic obtained from $\mathbf{H 1}$ or $\mathbf{H 2}$ by adding a set of Sahlqvist formulas as axioms. Then, $\mathbf{L}$ is characterized by a class of enlarged $\mathbf{B} . \mathbf{C}_{\square \diamond-\text { frames. }}$

The point of this theorem is that the $\operatorname{logic} \mathbf{L}$ is $\mathscr{D}^{\star}$-elementary, that is, there exists a set $\Phi$ of first-order sentences in the predicates $O, R, S_{\square}, S_{\diamond},{ }^{*}$, and the constants $e, u$ such that, for every descriptive $\mathbf{L}$-frame or enlarged $\mathbf{L}$-frame $\mathfrak{F}, \mathfrak{F}$ is an $\mathbf{L}$-frame iff $\mathfrak{F}$ satisfies each sentence in $\Phi$. Because the logics $\mathbf{H 1}$ and $\mathbf{H 2}$ are $\mathscr{D}^{\star}$-elementary, we are interested in showing that the logic $\mathbf{L}$ is $\mathscr{D}^{\star}$-elementary. In other words, we want to specify what qualifies as a first-order sentence corresponding to a given Sahlqvist formula. Following [7], we will sketch this construction. Although, essentially, we use the notion of general frames, we present some additional notions that are required for our discussion. For precise definitions of general frames and descriptive frames, see [7].

Below, $A\left[p_{1}, \ldots, p_{n}\right]$ denotes a formula $A$ whose variables are listed among $p_{1}, \ldots, p_{n}$. Let $\left\langle O, W, R, S_{\square}, S_{\diamond},{ }^{*}, e, u\right\rangle$ be an enlarged $\mathbf{L}$-frame. For $A\left[p_{1}, \ldots\right.$, $\left.p_{n}\right] \in \mathrm{Wff}$ and $X_{1}, \ldots, X_{n} \subseteq W, A\left[X_{1}, \ldots, X_{n}\right]$ is defined as follows:

- for $p_{i} \in \operatorname{Prop}, p_{i}\left[X_{1}, \ldots, X_{n}\right]=X_{i}$,

- $(B \wedge C)\left[X_{1}, \ldots, X_{n}\right]=B\left[X_{1}, \ldots, X_{n}\right] \cap C\left[X_{1}, \ldots, X_{n}\right]$,

- $(B \vee C)\left[X_{1}, \ldots, X_{n}\right]=B\left[X_{1}, \ldots, X_{n}\right] \cup C\left[X_{1}, \ldots, X_{n}\right]$,

- $(B \rightarrow C)\left[X_{1}, \ldots, X_{n}\right]=\left\{a \in W \mid \forall b, c \in W\left(R a b c \& b \in B\left[X_{1}, \ldots\right.\right.\right.$, $\left.\left.\left.X_{n}\right] \Rightarrow c \in C\left[X_{1}, \ldots, X_{n}\right]\right)\right\}$,

- $(\sim B)\left[X_{1}, \ldots, X_{n}\right]=\left\{a \in W \mid a^{*} \notin B\left[X_{1}, \ldots, X_{n}\right]\right\}$,

- $(\square B)\left[X_{1}, \ldots, X_{n}\right]=\left\{a \in W \mid \forall b \in W\left(S_{\square} a b \Rightarrow b \in B\left[X_{1}, \ldots, X_{n}\right]\right)\right\}$,

- $(\diamond B)\left[X_{1}, \ldots, X_{n}\right]=\left\{a \in W \mid \exists b \in W\left(S_{\diamond} a b \& b \in B\left[X_{1}, \ldots, X_{n}\right]\right)\right\}$.

For $a \in W$ and a nonnegative integer $n$, we write $a \uparrow^{n}=\left\{b \in W \mid S^{n} a b\right\}$. The frame-theoretic term $a_{1} \uparrow^{n_{1}} \cup \cdots \cup a_{k} \uparrow^{n_{k}}$, with (not necessarily distinct) $a_{1}, \ldots$, $a_{k} \in W$, is called an $S \mathbf{\square}$-term for brevity.

It suffices to consider a conjunction $\mathbf{\square}^{k}(B \rightarrow C)$ of a formula equivalent to the given Sahlqvist formula $A$. Let $\mathfrak{F}=\left\langle O, W, R, S_{\square}, S_{\diamond},{ }^{*}, e, u, P\right\rangle$ be a descriptive B.C $\square \diamond$-frame or an enlarged B.C $\square \diamond$-frame. Then, the following statements are mutually equivalent:

1. $(\mathfrak{F}, a) \models \boldsymbol{\square}^{k}\left(B\left[p_{1}, \ldots, p_{n}\right] \rightarrow C\left[p_{1}, \ldots, p_{n}, q_{1}, \ldots, q_{l}\right]\right)$,

2. $\forall X_{1}, \ldots, X_{n}, Y_{1}, \ldots, Y_{l} \in P\left(a \in \mathbf{\square}^{k}(B \rightarrow C)\left[X_{1}, \ldots, X_{n}, Y_{1}, \ldots, Y_{l}\right]\right)$,

3. $\forall X_{1}, \ldots, X_{n}, Y_{1}, \ldots, Y_{l} \in P \forall b_{1}, b_{2}, b_{3} \in W\left(S_{a}^{k} a b_{1} \& R b_{1} b_{2} b_{3} \&\right.$ $\left.b_{2} \in B\left[X_{1}, \ldots, X_{n}\right] \Rightarrow b_{3} \in C\left[X_{1}, \ldots, X_{n}, Y_{1}, \ldots, Y_{l}\right]\right)$;

4. $\forall X_{1}, \ldots, X_{n}, Y_{1}, \ldots, Y_{l} \in P \forall b_{1}, b_{2}, b_{3}, b_{4}, \ldots, b_{t} \in W\left(S_{\text {q }}^{k} a b_{1} \&\right.$ $R b_{1} b_{2} b_{3} \& D \& \bigwedge_{i \leq n} T_{i} \subseteq X_{i} \& \bigwedge_{j \leq m} c_{j} \in N_{j}\left[X_{1}, \ldots, X_{n}\right] \&$ $\left.\bigwedge_{h \leq l} u \in Y_{h} \Rightarrow b_{3} \in C\left[X_{1}, \ldots, X_{n}, Y_{1}, \ldots, Y_{l}\right]\right)$, where $D$ is a conjunction 
of formulas of the form $S \triangleleft b c, T_{i}$ are suitable $S_{\boldsymbol{\varpi}}$-terms, and $N_{j}\left[p_{1}, \ldots, p_{n}\right]$ are negative formulas;

5. $\forall b_{1}, \ldots, b_{t} \in W\left(S_{\mathbf{n}}^{k} a b_{1} \& R b_{1} b_{2} b_{3} \& D \Rightarrow \bigvee_{j \leq m+1} d_{j} \in K_{j}\left[T_{1}, \ldots, T_{n}\right.\right.$, $u \uparrow 0, \ldots, u \uparrow[0]$ ), where $d_{j}=c_{j}{ }^{*}$ for $j \leq m, K_{j}$ is a positive formula such that $N_{j}$ is equivalent to $\sim K_{j}$ for $j \leq m, d_{m+1}=b_{3}$, and $K_{m+1}$ is $C$.

Thus, the first-order sentence corresponding to $\mathbf{\square}^{k}(B \rightarrow C)$ can be written as

$$
\begin{aligned}
\forall a \in O \forall b_{1}, \ldots, b_{t} \in W & \left(S_{\mathbf{m}}^{k} a b_{1} \& R b_{1} b_{2} b_{3} \& D\right. \\
& \left.\Rightarrow \bigvee_{j \leq m+1} d_{j} \in K_{j}\left[T_{1}, \ldots, T_{n}, u \uparrow^{0}, \ldots, u \uparrow \mathbf{0}\right]\right) .
\end{aligned}
$$

\section{Halldén Completeness of $\mathrm{H} 2$}

From the discussion in Section 6, we have the strengthened result of Proposition 3.2.

Lemma 8.1 $\mathbf{H 2}$ is characterized by a strongly elementary class of completely serial $\mathbf{H 2}$-frames.

By Lemma 8.1 and Theorem 5.3, we have the following.

\section{Theorem 8.2 $\mathbf{H} 2$ is Halldén complete.}

Next, we consider the Halldén completeness of H2-logics with some Sahlqvist axioms. Because H2-frames are completely serial, so are the frames for H2-logics with any Sahlqvist axioms. We will also consider a class of frames in the usual sense, that is, when enlarged frames are not assumed, and determine for which logics they are strongly elementary. In light of Theorem 5.3, we hope to find Sahlqvist formulas that ensure that a class of frames for the H2-logic is strongly elementary. Since neither $e$ nor $u$ appear in our frame postulates, we do not assume the existence of $u$. Therefore, all propositional variables appearing in $C$ also appear in $B$; that is, $\operatorname{Var}(C) \subseteq \operatorname{Var}(B)$.

If $m>0$ in $(*)$, then the succedent is a disjunctive form that, in general, cannot be written by a conjunctive form. Thus, we may assume that $m=0$, which means that there is no subformula with the form of a negative formula in $B$. Then, $(*)$ can be simplified to

$$
\begin{gathered}
\forall a \in O \forall b_{1}, \ldots, b_{t} \in W\left(S_{\square}^{k} a b_{1} \& R b_{1} b_{2} b_{3} \& D\right. \\
\left.\Rightarrow d \in C\left[T_{1}, \ldots, T_{n}\right]\right) .
\end{gathered}
$$

Note that $K_{1}$ is just $C$ in $(*)$.

However, the succedent in $(* *)$ may still be a disjunctive form. For example, we consider the case $d \in p\left[b \uparrow_{\square}^{1} \cup c \uparrow_{\square}^{2}\right]$, that is, $S_{\square} b d$ or $S_{\square}^{2} c d$. It is impossible to write this with a conjunctive form. To avoid this situation, we may assume that $S_{\boldsymbol{\varpi}}$-terms are restricted to the form $c^{h} \boldsymbol{n}$, which means that each propositional variable in $B$ appears only once.

Furthermore, considering the definitions of $C\left[T_{1}, \ldots, T_{n}\right]$, we may assume that $C$ is of the form $\square^{l} C^{\prime}$, where $C^{\prime}$ is constructed using no connectives other than $\wedge$ and $\diamond$. Then, $(* *)$ can be simplified to

$$
\begin{gathered}
\forall a \in O \forall b_{1}, \ldots, b_{t}, d^{\prime} \in W\left(S_{\square}^{k} a b_{1} \& R b_{1} b_{2} b_{3} \& D \& S_{\square}^{l} d d^{\prime}\right. \\
\left.\Rightarrow d^{\prime} \in C^{\prime}\left[T_{1}, \ldots, T_{n}\right]\right) .
\end{gathered}
$$


Note here that $b_{i}=d$ for some $i(1 \leq i \leq t)$ and that the succedent is a conjunctive form.

We will examine some Sahlqvist formulas, called PC-Sahlqvist formulas, based on the above consideration. A formula $A$ is called $P C$-Sahlqvist if it is a conjunction of the form $\boldsymbol{\square}^{k}\left(B \rightarrow \mathbf{\square}^{l} C\right)$, where

- $k, l \geq 0$,

- $\operatorname{Var}(C) \subseteq \operatorname{Var}(B)$,

- $B$ is constructed from strongly positive formulas by applying $\wedge$ and $\diamond$, and no propositional variable occurs twice in $B$, and

- $C$ is constructed using no connectives other than $\wedge$ and $\diamond$

From Proposition 7.1 and the above observation, we have the following.

\section{Lemma 8.3}

(1) For any PC-Sahlqvist formula A, there exists a first-order formula $\varphi(a)$ in the predicates $O, R, S_{\square}, S_{\diamond}$, and ${ }^{*}$ having a as its only free variable, and such that the following holds for every descriptive $\mathbf{H 2}$-frame or $\mathbf{H 2}$-frame $\mathfrak{F}$ and every $a \in W:(\mathfrak{F}, a) \models A$ iff $\mathfrak{F}$ satisfies $\varphi(a)$.

(2) Let $\mathbf{L}$ be a logic obtained from $\mathbf{H} 2$ by adding a set of PC-Sahlqvist formulas as axioms. Then, $\mathbf{L}$ is characterized by a strongly elementary class of

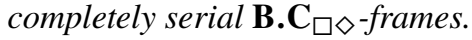

By Lemma 8.3 and Theorem 5.3, Theorem 8.2 can be extended to any logic obtained from $\mathbf{H} \mathbf{2}$ by adding any set of PC-Sahlqvist formulas as axioms.

Theorem 8.4 Every logic obtained from $\mathbf{H 2}$ by adding any set of PC-Sahlqvist formulas as axioms is Halldén complete.

As all axioms in Meyer-Fuhrmann schemes, except for (B15) and (Q2), are PCSahlqvist formulas, all Meyer-Fuhrmann logics with (D2) are regarded as special cases of $\mathbf{H 2}$ with PC-Sahlqvist axioms. Furthermore, the dual of (C2) is a PCSahlqvist formula. Thus, we have the following.

Corollary 8.5 (see [4]) If $\mathbf{L}$ is a logic constructed by adding (only) zero or more of the Meyer-Fuhrmann schemes to $\mathbf{R C}+\{(\mathrm{C} 2),(\mathrm{D} 2)\}$, then $\mathbf{L}$ is Halldén complete.

\section{Halldén Completeness of Classical Modal Logics}

We now consider the classical modal logics. If we adopt the enlarged frames introduced in Section 7, then it is hard to prove the enlarged version of Lemma 5.1 because of clause (ep3). Thus, instead of using enlarged frames, we will consider the usual frames, in the sense of Routley-Meyer semantics, to prove the Halldén completeness of the classical modal logics. In the following discussion, frames refer to those in the sense of Routley-Meyer semantics.

The nonnormal classical modal logic EMC, where $\mathbf{E M C}=\mathbf{R C}+\{(\mathrm{C} 1)\}$, is not complete with respect to any class of EMC-frames. In fact, $\square(A \vee \sim A)$ is not a theorem of EMC (see Chellas [1, p. 251]), though it is valid in any EMC-frame. Therefore, it is impossible to consider the Halldén completeness of EMC (and its modal extensions) in our framework.

In contrast, Proposition 3.2, which shows Kripke completeness in the usual Routley-Meyer semantics, also holds for several normal classical modal logics, including $\mathbf{K}$ and $\mathbf{K D}$, where $\mathbf{K D}=\mathbf{K}+\{(\mathrm{D} 2)\}$. As mentioned in Section $6, \mathbf{K}$ is 
not Halldén complete. This fact is related to the problem of canonical models; that is, nondegenerate canonical modeling is required for $\mathbf{K}$. On the other hand, from the fact that (B1), (B2), and (D2) are theorems of KD and a similar argument in Lemma 8.3, we have the following.

\section{Lemma 9.1}

(1) KD is characterized by a strongly elementary class of completely serial KDframes.

(2) Let $\mathbf{L}$ be a logic obtained from $\mathbf{K D}$ by adding a set of PC-Sahlqvist formulas as axioms. Then, $\mathbf{L}$ is characterized by a strongly elementary class of

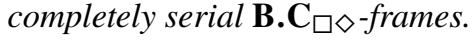

By Lemma 9.1 and Theorem 5.3, we have the following.

Theorem 9.2 The classical modal logic KD, and all logics obtained from KD by adding any set of PC-Sahlqvist formulas as axioms, are Halldén complete.

Note that, as long as $e$ and $u$ are neglected, the frames for $\mathbf{K}$ are exactly equal to the usual Kripke frames for $\mathbf{K}$ because the following holds in every $\mathbf{K}$-frame: (1) $O=W$, (2) Rabc implies $a=b=c$, and (3) $a^{*}=a$ (for more information, see [7, p. 408]). Thus, Theorem 9.2 can also be proven in the sense of usual Kripke frames.

\section{References}

[1] Chellas, B. F., Modal Logic: An Introduction, Cambridge University Press, 1980. Zbl 0431.03009. MR 0556867. 348

[2] Mares, E. D., "The semantic completeness of RK," Reports on Mathematical Logic, vol. 26 (1992), pp. 3-10. Zbl 0804.03013. MR 1231308. 344

[3] Mares, E. D., "Classically complete modal relevant logics," Mathematical Logic Quarterly, vol. 39 (1993), pp. 165-77. Zbl 0804.03012. MR 1269906. DOI 10.1002/malq.19930390119. 344

[4] Mares, E. D., "Halldén-completeness and modal relevant logic," Logique et Analyse (N. S.), vol. 181 (2003), pp. 59-76. Zbl 1069.03012. MR 2102337. 333, 334, 339, 340, 343, 344, 348

[5] Routley, R., and R. K. Meyer, "The semantics of entailment, III," Journal of Philosophical Logic, vol. 1 (1972), pp. 192-208. Zbl 0317.02018. MR 0409116. 333

[6] Routley, R., V. Plumwood, R. K. Meyer, and R. T. Brady, Relevant Logics and Their Rivals, I, Ridgeview Publishing Company, Atascadero, Calif., 1982. MR 0728950. 333, 334, 342

[7] Seki, T., "A Sahlqvist theorem for relevant modal logics," Studia Logica, vol. 73 (2003), pp. 383-411. Zbl 1027.03020. MR 1977820. DOI 10.1023/A:1023335229747. 334, $338,342,345,346,349$

[8] Seki, T., "General frames for relevant modal logics," Notre Dame Journal of Formal Logic, vol. 44 (2003), pp. 93-109. Zbl 1071.03009. MR 2060057. DOI 10.1305/ndjfl/1082637806. 339

[9] Seki, T., "Completeness of relevant modal logics with disjunctive rules," Reports on Mathematical Logic, vol. 44 (2009), pp. 3-18. Zbl 1170.03011. MR 2531527. 338

[10] van Benthem, J. F. A. K., and I. L. Humberstone, "Halldén-completeness by gluing of Kripke frames," Notre Dame Journal of Formal Logic, vol. 24 (1983), pp. 426-30. Zbl 0487.03008. MR 0717904. DOI 10.1305/ndjfl/1093870446. 333 


\section{Acknowledgments}

The author would like to thank the anonymous referees for their useful comments. This work was partially supported by Grant for Promotion of Niigata University Research Project 23C050.

University Evaluation Center, Headquarters for Strategy and Planning Niigata University

8050 Ikarashi 2-no-cho

Nishi-ku, Niigata City 950-2181

Japan

tseki@adm.niigata-u.ac.jp 